Research Article

\title{
Incorporating a Patient Dichotomous Characteristic in Cancer Phase I Clinical Trials Using Escalation with Overdose Control
}

\author{
Mourad Tighiouart, Galen Cook-Wiens, and André Rogatko \\ Biostatistics and Bioinformatics Research Center, Samuel Oschin Comprehensive Cancer Institute, \\ 8700 Beverly Boulevard, PACT, Suite 900C, Los Angeles, CA 90048, USA \\ Correspondence should be addressed to Mourad Tighiouart, mourad.tighiouart@cshs.org
}

Received 29 June 2012; Accepted 5 September 2012

Academic Editor: Yichuan Zhao

Copyright (C) 2012 Mourad Tighiouart et al. This is an open access article distributed under the Creative Commons Attribution License, which permits unrestricted use, distribution, and reproduction in any medium, provided the original work is properly cited.

\begin{abstract}
We describe a design for cancer phase I clinical trials that takes into account patients heterogeneity thought to be related to treatment susceptibility. The goal is to estimate the maximum tolerated dose (MTD) given patient's specific dichotomous covariate value. The design is Bayesian adaptive and is an extension of escalation with overdose control (EWOC). We will assess the performance of this method by comparing the following designs via extensive simulations: (1) design using a covariate; patients are accrued to the trial sequentially and the dose given to a patient depends on his/her baseline covariate value, (2) design ignoring the covariate; patients are accrued to the trial sequentially and the dose given to a patient does not depend on his/her baseline covariate value, and (3) design using separate trials; in each group, patients are accrued to the trial sequentially and EWOC is implemented in each group. These designs are compared with respect to safety of the trial and efficiency of the estimates of the MTDs via extensive simulations. We found that ignoring a significant baseline binary covariate in the model results in a substantial number of patients being overdosed. On the other hand, accounting for a nonsignificant covariate in the model has practically no effect on the safety of the trial and efficiency of the estimates of the MTDs.
\end{abstract}

\section{Introduction}

The main objective of cancer phase I clinical trials is to determine a maximum tolerated dose (MTD) of a new experimental drug or combination of known drugs for use in a phase II trial. These trials enroll advanced stage cancer patients who have exhausted all standard therapies sequentially in cohorts of size one or more patients and dose level assignment to a given cohort of patients is dependent upon the dose levels and toxicity outcomes of the previously treated cohorts of patients. Adaptive statistical designs for cancer phase I clinical trials have been studied extensively in the last two decades, see for example, O'Quigley et al. 
[1], Durham and Flournoy [2], Korn et al. [3], Whitehead [4], Babb et al. [5], Gasparini and Eisele [6], Mukhopadhyay [7], and Haines et al. [8]. See also Ting [9] and Chevret [10] for a more comprehensive review of these statistical designs.

A key assumption implied by the definition of the phase I target dose (MTD) is that every subgroup of the patient population has the same MTD. That is, it is assumed that the patient population is homogeneous in terms of treatment tolerance and every patient should be treated at the same dose. As a result, no allowance is made for individual patient differences in susceptibility to treatment. Recent progress in our understanding of pharmacokinetics and the genetics of drug metabolism has led to the development of new strategies of drug allocation that accommodate individual patient needs, see [11-13]. For example, Newell [14] showed how impaired renal function can result in reduced clearance of carboplatin and a dosing formulae based on renal function was developed. In this paper, we present design operating characteristics of a design proposed by Babb et al. [5] known as escalation with overdose control (EWOC) by accounting for patients heterogeneity thought to be related to treatment susceptibility. In the case of a binary covariate, we will assess the performance of this method by comparing the following designs via extensive simulations: (1) design using a covariate; patients are accrued to the trial sequentially and the dose given to a patient depends on his/her covariate value, (2) design ignoring the covariate; patients are accrued to the trial sequentially and the dose given to a patient does not depend on his/her covariate value, and (3) design using separate trials; in each group, patients are accrued to the trial sequentially and EWOC is implemented in each group. O'Quigley et al. [15] investigated the performance of a two-stage continual reassessment method (CRM) using a binary covariate. They considered 3 different models for the dose-toxicity relationship and maximum likelihood method was used to estimate the model parameters. This required starting the escalation scheme using some ad hoc mechanism until the first toxicity is observed. They found that significant gains can be made using the two-sample $\mathrm{CRM}$ when there are group imbalances. However, there may not be enough patients in one group to detect that effect. O'Quigley and Paoletti [16] considered a two-group CRM design incorporating ordering of the two groups with respect to treatment tolerability in designing a phase I trial. Babb and Rogatko [17] extended EWOC to allow the utilization of information concerning individual patient differences in susceptibility to treatment. This was applied to a trial involving patients with advanced adenocarcinomas of gastrointestinal origin treated with PNU-214565 (PNU). PNU is a murine Fab fragment of the monoclonal antibody 5T4 fused to a mutated superantigen staphylococcal enterotoxin A (SEA). Preclinical testing demonstrated that the action of PNU is moderated by the neutralizing capacity of antiSEA antibodies. Consequently, dose levels were adjusted during the trial according to each patient's pretreatment plasma concentration of anti-SEA antibodies. However, design operating characteristics were not studied.

This paper is organized as follows. Section 2 describes the model likelihood and prior distributions and the conduct of the trial using EWOC scheme for three different designs. We present some simulation results in Section 3 and concluding remarks are presented in Section 4.

\section{Method}

\subsection{Model}

In this section, we describe a Bayesian adaptive design which accounts for patient heterogeneity thought to be related to treatment susceptibility. Let $X_{\min }$ and $X_{\max }$ denote 
the minimum and maximum dose levels available for use in the trial. Clinicians choose these levels in the belief that $X_{\min }$ is safe when administered to humans and $X_{\max }$ is too toxic, see [18] how these levels were selected for a real prospective trial. Denote by $Z$ the observable baseline binary covariate taking values 0 or 1 and let

$$
P_{z}(x)=\operatorname{Prob}(\mathrm{DLT} \mid \text { Dose }=x, Z=z),
$$

be the probability of dose limiting toxicity (DLT) for a patient with baseline covariate $z$ and treated with dose level $x$. For simplicity, we consider the logistic model to describe the dosetoxicity relation

$$
P_{z}(x)=\frac{\exp \left(\beta_{0}+\beta_{1} x+\eta z\right)}{1+\exp \left(\beta_{0}+\beta_{1} x+\eta z\right)}
$$

We assume that $\beta_{1} \geq 0$ so that $P_{z}(x)$ is an increasing function of dose $x$. This is a reasonable assumption for cytotoxic agents. Model (2.2) implies a constant odds ratio of toxicity between the two groups of patients in the sense that this odds ratio does not depend on the dose level.

The MTD for a patient with covariate value $z$ is defined as the dose $\gamma(z)$ that results in a probability equal to $\theta(z)$ that a DLT will manifest within one cycle of therapy. The value chosen for the target probability of DLT $\theta(z)$ would depend on the nature and consequences of the dose-limiting toxicity; it would be set relatively high when the DLT is a transient, correctable or nonfatal condition, and low when it is life threatening or lethal [5]. We will assume that $\theta(z)$ is constant in $z$ although the methodology can be adapted to different target probabilities of toxicities. In practice, clinicians use a constant target probability of DLT $\theta$ since we do not know a priori how the treatment under study affects the different groups of patients defined by their baseline covariate value. It follows from the dose-toxicity model (2.2) that the MTD is

$$
\gamma(z)=\frac{1}{\beta_{1}}(\operatorname{logit}(\theta)-\alpha-\eta z) .
$$

Let $\rho_{0, z}$ be the probability of DLT at the initial dose given to a patient with covariate value $z$. In the statistical design of a phase I clinical trial, it is convenient to specify the prior distribution on parameters the clinicians can easily interpret. For instance, Babb and Rogatko [17] reparameterized model (2.2) in terms of the MTD associated with the maximumanticipated plasma concentration of anti-SEA antibodies and the probabilities of DLT when the minimum allowable dose is administered to patients with pretreatment anti-SEA concentrations selected to span the range of this covariate. Here, we reparameterize model (2.2) in terms of $\gamma_{0}=\gamma(0), \gamma_{1}=\gamma(1)$, and $\rho_{0,0}$. We chose this reparameterization because the MTDs for each group are the parameters of interest. However, other parameterizations such 
as difference between the MTDs in both groups are possible. Using the definition of the MTDs and probability of toxicity at the initial dose $x_{1}$, one can show that

$$
\begin{gathered}
\beta_{0}=\frac{1}{\left(\gamma_{0}-x_{1}\right)}\left[\gamma_{0} \operatorname{logit}\left(\rho_{0,0}\right)-x_{1} \operatorname{logit}(\theta)\right], \\
\beta_{1}=\frac{1}{\left(\gamma_{0}-x_{1}\right)}\left[\operatorname{logit}(\theta)-\operatorname{logit}\left(\rho_{0,0}\right)\right], \\
\eta=\frac{\left(\gamma_{0}-\gamma_{1}\right)}{\left(\gamma_{0}-x_{1}\right)}\left[\operatorname{logit}(\theta)-\operatorname{logit}\left(\rho_{0,0}\right)\right] .
\end{gathered}
$$

We note that the probability of DLT does not depend on the parameter $\gamma_{1}$ when $z=0$. Denote by $p\left(\rho_{0,0}, \gamma_{0}, \gamma_{1}, x_{1}\right)$ and $p\left(\rho_{0,0}, \gamma_{0}, x_{0}\right)$ the probabilities of DLT for a patient with covariate value 1 and 0 , respectively. These probabilities are obtained using the dose-toxicity model (2.2) with $\beta_{0}, \beta_{1}, \eta$ given by (2.4).

\subsection{Likelihood}

Suppose that after the $l$ th patient with baseline covariate value $z$ is treated with dose $x_{z, l}$, there are $m_{l}$ patients with covariate value $z=0$ and $k_{l}$ patients with covariate value $z=1$. Let $y_{z, l}$ be the toxicity outcome ( 1 for DLT and 0 for no DLT) for the $i$ th patient with covariate value $z$. The likelihood of the data is

$$
\begin{aligned}
L\left(\rho_{0,0}, \gamma_{0}, \gamma_{1} \mid D_{l}\right)= & \prod_{i=1}^{m_{l}}\left[p\left(\rho_{0,0}, \gamma_{0}, x_{0, i}\right)^{y_{0, i}}\left(1-p\left(\rho_{0,0}, \gamma_{0}, x_{0, i}\right)\right)^{1-y_{0, i}}\right] \\
& \times \prod_{j=1}^{k_{l}}\left[p\left(\rho_{0,0}, \gamma_{0}, \gamma_{1}, x_{1, i}\right)^{y_{1, i}}\left(1-p\left(\rho_{0,0}, \gamma_{0}, \gamma_{1}, x_{1, i}\right)\right)^{1-y_{1, i}}\right],
\end{aligned}
$$

where $D_{l}=\left\{\left(x_{0,1}, y_{0,1}\right), \ldots,\left(x_{0, m_{l}}, y_{0, m_{l}}\right),\left(x_{1,1}, y_{1,1}\right), \ldots,\left(x_{1, k_{l}}, y_{0, k_{l}}\right)\right\}$ and $m_{l}+k_{l}=l$.

Let $h\left(\rho_{0,0}, \gamma_{0}, \gamma_{1}\right)$ be a prior distribution on the parameters $\rho_{0,0}, \gamma_{0}$, and $\gamma_{1}$. The posterior distribution is

$$
\pi\left(\rho_{0,0}, \gamma_{0}, \gamma_{1} \mid D_{l}\right)=c\left(D_{l}\right) L\left(\rho_{0,0}, \gamma_{0}, \gamma_{1} \mid D_{l}\right) h\left(\rho_{0,0}, \gamma_{0}, \gamma_{1}\right),
$$

where $c\left(D_{l}\right)$ is a normalizing constant. This joint posterior is clearly intractable and WinBugs and a Markov chain Monte Carlo sampler will be devised to estimate features of this joint posterior distribution as in Tighiouart et al. [18].

\subsection{Prior Distributions}

Another advantage of the reparameterization in (2.4) is the natural specification of vague but proper prior densities for the model parameters. Indeed, under the assumption that $\gamma_{0}, \gamma_{1}$ belong to $\left[X_{\min }, X_{\max }\right]$ with prior probability 1 and no prior assumptions on whether one group can tolerate higher doses better than the other, we can take $\left(\gamma_{0}, \gamma_{1}\right) \sim$ Uniform 
$\left[X_{\min }, X_{\max }\right]^{2}$ and $\gamma_{0}$ independent of $\gamma_{1}$. If on the other hand, we have a priori belief that one group can tolerate higher doses better than the other group for example, then $\left(\gamma_{0}, \gamma_{1}\right)$ can be taken to be uniform on the triangle $X_{\min }<\gamma_{0}<\gamma_{1}<X_{\max }$. Design operating characteristics should be performed when designing prospective trials when considering informative priors. The prior distribution for $\rho_{0,0}$ is taken as a uniform in $[0, \theta]$, which reflects a lack of prior knowledge regarding the probability of DLT at the initial dose.

\subsection{Trial Design}

Denote by $A$ and $B$ the two groups of patients corresponding to covariate values 0 and 1 , respectively. We assume that the support of the MTDs $\gamma_{0}$ and $\gamma_{1}$ are contained in $\left[X_{\min }, X_{\max }\right]$. That is, we assume that dose levels $X_{\min }$ and $X_{\max }$ are identified a priori such that $\gamma_{0}, \gamma_{1}$ belong to $\left[X_{\min }, X_{\max }\right]$ with prior (and hence posterior) probability 1 . We note that if the prior distribution $\pi\left(\gamma_{1}\right)$ is independent of the joint prior distribution of $\left(\rho_{0,0}, \gamma_{0}\right)$, then $\pi\left(\gamma_{1}\right)$ is never updated unless a patient in group $B$ is enrolled in the trial. In the case of such priors, the trial proceeds as follows.

The first patient in either group receives the dose $x_{1}=X_{\min }$. Let $\Pi_{z, 1}$ be the marginal posterior cdf of the MTD $\gamma_{z}, z=0,1$. Suppose that the first patient belongs to group $A$. If the second patient belongs to group $A$, then he or she will receive the dose $x_{0,2}=\Pi_{0,1}^{-1}(\alpha)$ so that the posterior probability of exceeding the MTD $\gamma_{0}$ is equal to the feasibility bound $\alpha$. If the second patient belongs to group $B$, then he or she will receive the dose $x_{1}=X_{\min }$. In general, the first time a patient is assigned to a given group always receives $x_{1}=X_{\min }$ no matter how many patients have been enrolled in the other group. Once $l$ patients have been enrolled in the trial with at least one patient treated in each group, the $l+1$-st patient with covariate value $z$ receives the dose $x_{z, l+1}=\Pi_{z, l}^{-1}(\alpha)$. The trial proceeds until a total of $n$ patients have been accrued. At the end of the trial, we estimate the MTD as $\widehat{\gamma}_{z}=\Pi_{z, n}^{-1}(\alpha), z=0,1$.

\section{Simulation Studies}

\subsection{Comparison of Three Designs}

In order to assess the operating characteristics of this design when designing a prospective trial, we explored the behavior of this method when we adjust for a significant covariate. We also evaluated the performance of this design when adjusting for a nonsignificant baseline covariate. Finally, its performance was also explored when two parallel trials are used instead of adjusting for a binary baseline covariate. Therefore, we study design operating characteristics by comparing the following designs.

(i) Design using a covariate; patients are accrued to the trial sequentially and the dose given to a patient is calculated assuming model (2.2).

(ii) Design ignoring the covariate; patients are accrued to the trial sequentially and the dose given to a patient is calculating assuming model (2.2) without the covariate, that is, as in the original EWOC.

(iii) Design using separate trials; in each group, patients are accrued to the trial sequentially and EWOC is implemented in each group.

Comparisons will be carried out under several scenarios for the true values of the MTDs $\gamma_{0}$ and $\gamma_{1}$. Since the main goal of cancer phase I clinical trials is to efficiently estimate the MTD 
while protecting patients from potentially toxic side effects, we will assess the safety of the trial and efficiency of the estimate of the MTDs by simulating a large number of trials $M$ under each model and compare the proportion of patients exhibiting DLT, the average bias bias $_{\text {ave }}=M^{-1} \sum_{i=1}^{M} \widehat{\gamma}_{z, i}-\gamma_{z \text {,true }}$ and the estimated mean square error MSE $=M^{-1} \sum_{i=1}^{M}\left(\widehat{\gamma}_{z, i}-\right.$ $\left.\gamma_{z, \text { true }}\right)^{2}$, where $\hat{\gamma}_{z, i}$ is the MCMC estimate of the Bayes estimate of the marginal posterior distribution of the MTD at the end of the $i$ th trial, $z=0,1$. In addition, the models are further compared with respect to the proportion of patients that were overdosed. Here, a patient with baseline covariate $z$ is overdosed if this patient has been given a dose $x$ such that $x>$ $x^{*}$, where $x^{*}$ is defined as the dose for which $P\left(\right.$ DLT $\left.\mid x^{*}, z\right)=\theta+0.05$. This probability is calculated using the parameter values from the corresponding scenario.

\subsection{Simulation Setup}

The simulation results presented below all assume that the feasibility bound $\alpha=0.25$ and that the dose levels are standardized so that the starting dose $x_{1}$ equals to the minimum dose for each trial $X_{\min }=0$ and all subsequent dose levels are selected from the unit interval. The target probability of DLT is fixed at $\theta=0.33, \rho_{0,0}=0.05$, and the total sample size is $n=42$. We consider several scenarios corresponding to combinations of four possible values of $\gamma_{0}, \gamma_{1}, 0.2,0.4,0.6$, and 0.8 . In all simulations, the prior distributions for $\rho_{0,0}, \gamma_{0}, \gamma_{1}$ were taken as uniform in $[0, \theta] \times\left[X_{\min }, X_{\max }\right]^{2}$ with $\rho_{0,0}, \gamma_{0}, \gamma_{1}$ independent a priori.

For design (1), a patient is randomly selected from either group $A$ or $B$ with equal probability so that the total number of patients in group $A, m$, equals to the total number of patients in group $B, k$. For each pair $\left(\gamma_{0}, \gamma_{1}\right)$ in $\{0.2,0.4,0.6,0.8\} \times\{0.2,0.4,0.6,0.8\}$, we simulate 1000 trials and calculate the proportion of patients that were overdosed, the proportion of patients exhibiting DLT, the average bias, and the estimated MSE. For design (2), the covariate for each patient is recorded but it is not taken into account when calculating the dose level for the next patient. Again, we simulate 1000 trials and calculate the proportion of patients that were overdosed, the proportion of patients exhibiting DLT, the average bias, and the estimated MSE. For the third design, separate trials are simulated in each group and the summary statistics are calculated based on 1000 simulated trials in each group. In all cases, the responses $y_{z, i}$ are generated from model (2.2).

\subsection{Results}

Table 1 gives the overall proportion of patients exhibiting DLT, the proportion exhibiting DLT in each group, the proportion of patients in each group that are overdosed, the bias, and MSE of the estimates of the MTDs when design (i) in Section 3.1 is used. Table 2 gives the summary statistics corresponding to the safety of the trial when design (ii) is used. The overall proportion of patients exhibiting DLT is always less than $\theta=0.33$ under all entertained scenarios and it is uniformly lower for a design which accounts for the baseline covariate relative to the design ignoring this covariate. The same conclusion holds when comparison of these two designs is carried out within each group. On the other hand, the proportion of patients being overdosed in group $A$ is much higher when the two groups of patients differ in their susceptibility to treatment and this difference is not taken into account. This proportion can be as high as $16 \%$ in the case where $\left(\gamma_{0}, \gamma_{1}\right)=(0.4,0.8)$. This is not surprising because when a difference in the MTDs is not taken into account in the model, then the sequence of doses generated by the design tends to cluster around a weighted average of 
Table 1: EWOC with Covariate. Design operating characteristic with respect to safety and efficiency of the trial.

\begin{tabular}{lcccccc}
\hline$\left(\gamma_{0}, \gamma_{1}\right)$ & $0.2,0.4$ & $0.2,0.6$ & $0.2,0.8$ & $0.4,0.6$ & $0.4,0.8$ & $0.6,0.8$ \\
\hline Proportion of DLTs & 0.3032 & 0.2735 & 0.2505 & 0.2442 & 0.2231 & 0.1954 \\
Proportion of DLTs in group A & 0.3058 & 0.2758 & 0.2495 & 0.2432 & 0.2230 & 0.1932 \\
Proportion of DLTs in group B & 0.3007 & 0.2713 & 0.2514 & 0.2451 & 0.2232 & 0.1975 \\
Proportion overdosed in group A & 0.5958 & 0.6236 & 0.6199 & 0.3174 & 0.3738 & 0.1029 \\
Proportion overdosed in group B & 0.0934 & 0.0448 & 0.0102 & 0.0373 & 0.0044 & 0.0019 \\
Bias $\left(\gamma_{1}\right)$ & -0.0090 & -0.0122 & -0.0174 & -0.0326 & -0.0432 & -0.0910 \\
Bias of $\left(\gamma_{2}\right)$ & -0.0585 & -0.1218 & -0.2185 & -0.1075 & -0.2014 & -0.2013 \\
MSE $\left(\gamma_{1}\right)$ & 0.0484 & 0.0501 & 0.0505 & 0.0916 & 0.0968 & 0.1476 \\
MSE $\left(\gamma_{2}\right)$ & 0.1068 & 0.1711 & 0.2622 & 0.1635 & 0.2451 & 0.2437 \\
\hline
\end{tabular}

Table 2: EWOC with no Covariate. Design operating characteristic with respect to safety of the trial.

\begin{tabular}{lcccccc}
\hline$\left(\gamma_{0}, \gamma_{1}\right)$ & $0.2,0.4$ & $0.2,0.6$ & $0.2,0.8$ & $0.4,0.6$ & $0.4,0.8$ & $0.6,0.8$ \\
\hline Proportion of DLTs & 0.3156 & 0.3172 & 0.3143 & 0.2824 & 0.2702 & 0.2370 \\
Proportion of DLTs in group A & 0.3170 & 0.3180 & 0.3105 & 0.2856 & 0.2737 & 0.2389 \\
Proportion of DLTs in group B & 0.3142 & 0.3164 & 0.3180 & 0.2791 & 0.2668 & 0.2350 \\
Proportion overdosed in group A & 0.6495 & 0.7415 & 0.775 & 0.4091 & 0.5298 & 0.1761 \\
Proportion overdosed in group B & 0.0184 & 0.0006 & 0.0000 & 0.0109 & 0.0000 & 0.0003 \\
\hline
\end{tabular}

the two "true" MTDs, where the weights depends on the number of patients in each group. If on the other hand, the models accounts for the difference in the MTDs, then the distribution of the sequence of doses is bimodal clustering around the two "true" MTDs, as displayed in Figure 1, which shows the histogram of all doses with fitted density (dashed line) when $\left(\gamma_{0}, \gamma_{1}\right)=(0.3,0.6)$. The difference in the proportion of patients being overdosed in group $B$ between the two designs is practically negligible. When the two MTDs are equal and the design does account for the baseline covariate, Tables 3 and 4 show that the proportions of DLTs (overall and within each group) is no more than the target probability of DLT $\theta$, and the differences in these proportions between the two designs are practically not important. No design is uniformly better than the other in terms of the proportion of patients being overdosed. For instance, when $\gamma_{0}=\gamma_{1}=0.2$, the proportion of patients in group $A$ is 0.336 when a covariate is used and this proportion is 0.275 when the covariate is not taken into account. On the other hand, when $\gamma_{0}=\gamma_{1}=0.4$, the proportion of patients in group $A$ is 0.179 when a covariate is used but this proportion is 0.21 when the covariate is not taken into account. In fact, these proportions are equal on the average across the four scenarios for the true value of the MTD $\gamma=\gamma_{0}=\gamma_{1}$. Tables 3 and 4 also show that the bias and MSE of the estimates of the MTD is higher when a nonsignificant covariate is used in the model, with the higher values obtained the true MTD is high, $\gamma=0.6,0.8$.

Table 5 gives the summary statistics when separate trials enrolling $n=21$ patients are used. As before, the proportion of patients exhibiting DLT does not exceed the target probability of DLT $\theta$. When the true MTDs are the same, the overall proportion of patients that are overdosed using a model with a baseline covariate (Table 3 ) is lower than the corresponding proportion if parallel trials were used (Table 5) when $\gamma=0.2,0.4$. The differences in these proportions for $\gamma=0.6,0.8$ are negligible. The bias of the estimate of the MTD is about the same for both designs. In the case where the MTDs $\gamma_{0}$ and $\gamma_{1}$ are 
Table 3: EWOC with Covariate. Design operating characteristic with respect to safety and efficiency of the trial.

\begin{tabular}{lcccc}
\hline$\gamma_{0}=\gamma_{1}$ & 0.2 & 0.4 & 0.6 & 0.8 \\
\hline Proportion of DLTs & 0.3337 & 0.2721 & 0.2183 & 0.1759 \\
Proportion of DLTs in group A & 0.3319 & 0.2751 & 0.2222 & 0.1764 \\
Proportion of DLTs in group B & 0.3356 & 0.2691 & 0.2143 & 0.1754 \\
Proportion overdosed in group A & 0.3361 & 0.1790 & 0.04995 & 0.0008 \\
Proportion overdosed in group B & 0.3346 & 0.1802 & 0.0497 & 0.0010 \\
Bias $\left(\gamma_{1}\right)$ & -0.0134 & -0.0387 & -0.0965 & -0.2002 \\
MSE $\left(\gamma_{1}\right)$ & 0.0716 & 0.1373 & 0.2142 & 0.3425 \\
\hline
\end{tabular}

Table 4: EWOC with no Covariate. Design operating characteristic with respect to safety and efficiency of the trial.

\begin{tabular}{lcccc}
\hline$\gamma_{0}=\gamma_{1}$ & 0.2 & 0.4 & 0.6 & 0.8 \\
\hline Proportion of DLTs & 0.3243 & 0.2939 & 0.2527 & 0.2109 \\
Proportion of DLTs in group A & 0.3251 & 0.2944 & 0.2515 & 0.2083 \\
Proportion of DLTs in group B & 0.3236 & 0.2934 & 0.2539 & 0.2134 \\
Proportion overdosed in group A & 0.2755 & 0.2108 & 0.0774 & 0.0023 \\
Proportion overdosed in group B & 0.2761 & 0.2050 & 0.0749 & 0.0026 \\
Bias $\left(\gamma_{1}\right)$ & -0.0119 & -0.0197 & -0.0537 & -0.1198 \\
MSE $\left(\gamma_{1}\right)$ & 0.0353 & 0.0684 & 0.1032 & 0.1537 \\
\hline
\end{tabular}

Table 5: EWOC. Design operating characteristic with respect to safety and efficiency of the trial.

\begin{tabular}{lcccc}
\hline$\gamma$ & 0.2 & 0.4 & 0.6 & 0.8 \\
\hline Proportion of DLTs & 0.3372 & 0.2778 & 0.2162 & 0.1737 \\
Proportion overdosed & 0.3684 & 0.2067 & 0.0417 & 0.0001 \\
Bias $(\gamma)$ & -0.0086 & -0.0249 & -0.08124 & -0.1915 \\
MSE $(\gamma)$ & 0.0464 & 0.0858 & 0.1381 & 0.2246 \\
\hline
\end{tabular}

Density curves

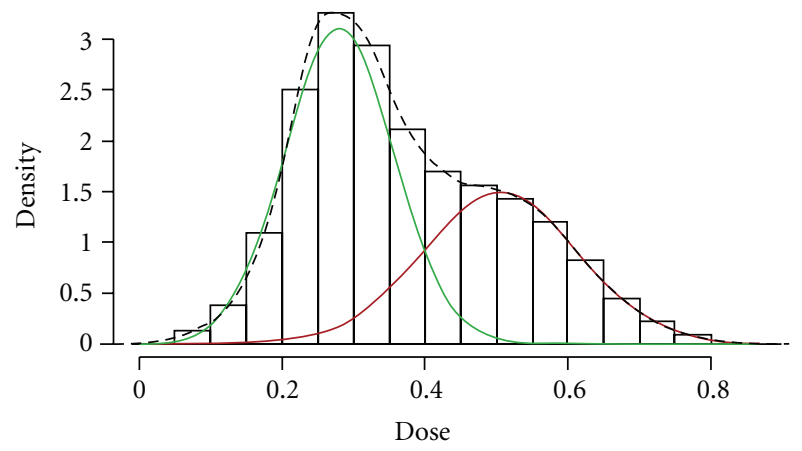

Figure 1: Histogram and fitted density (dashed line) of dose allocations for patients 2 through 80 based on 1000 simulated trials using the model with covariate. The true MTDs are $\gamma_{0}=0.3, \gamma_{1}=0.6$, and $\rho_{0}=$ $0.05, \theta=0.33, \alpha=0.25$. 
different, more patients are overdosed using a model with a baseline covariate compared to using parallel trials and the differences in the bias and MSE are negligible, see Tables 1 and 5.

Based on these results, we recommend adjusting for a baseline covariate thought to be related to treatment susceptibility when designing a cancer phase I trials whenever possible. We stand to lose little if we were to use a design with a covariate when in fact there is no difference between the MTDs of the two groups.

\section{Discussion}

We have presented design operating characteristics of a Bayesian adaptive design which accounts for a patient dichotomous baseline covariate using EWOC scheme. The design is suitable for cancer phase I clinical trials where the goal is to estimate the conditional MTD given patients' covariate value.

We have found that if the two MTDs are different and the design does not adjust for this heterogeneity, then the trial will result in more patients being overdosed. If the two MTDs are the same and the design adjusts for patients' heterogeneity, then slightly more patients can be overdosed if the true MTD is low relative to a design with no covariate but these proportions are equal on the average across the four scenarios for the true value of the MTD. Thus, we stand to lose little if we do include a statistically nonsignificant covariate in the model. Incidentally, this conclusion is in agreement with the findings in O'Quigley et al. [15]. We carried out other simulations (results not shown) for various sample sizes, allocation ratios, probability of DLT at the initial dose $\rho_{0}$. The results and conclusions were essentially the same. Ratain et al. [13] showed the importance of including patient's plasma concentration of anti-SEA antibodies in order to determine the MTD of the agent PNU-214565 as a function of this continuous baseline covariate. In a similar trial, Tighiouart and Rogatko [16] showed how more patients were overdosed when a baseline covariate, cancer type, was not accounted for in the model. Indeed, a retrospective analysis of a cancer phase I trial using a baseline continuous covariate showed that nonsmall cell cancer patients and pancreatic patients were treated at suboptimal doses whereas renal cell carcinoma patients were overdosed, with $36.4 \%$ experiencing DLT; the target probability of DLT was $\theta=0.2$. This last example is in agreement with the simulation results we obtained in this paper. We are in the process of determining model operating characteristics in the presence of a continuous covariate, more than one covariate, and interaction term. An important question is to decide whether or not to include patients' covariate values during the trial. Although the previous results seem to indicate that we stand to lose little in terms of the proportion of patients being overdoses and the efficiency of the estimate of the MTDs when covariate information is taken into account in the model when in fact, this covariate is not predictive of DLT, determining the value of this covariate may involve a monetary cost. This is the case when patients need to be genotyped and certain biomarker expressions need to be determined.

\section{Acknowledgments}

Supported in part by the National Center for Research Resources, Grant UL1RR033176, and is now at the National Center for Advancing Translational Sciences, Grant UL1TR000124 (M. Tighiouart and A. Rogatko), Grant UL1TR000124 (M. Tighiouart and A. Rogatko), Grant 5P01CA098912-05 (A. Rogatko) and P01 DK046763 (A. Rogatko). The content is solely the responsibility of the authors and does not necessarily represent the official views of the NIH. 


\section{References}

[1] J. O'Quigley, M. Pepe, and L. Fisher, "Continual reassessment method: a practical design for phase 1 clinical trials in cancer," Biometrics, vol. 46, no. 1, pp. 33-48, 1990.

[2] S. D. Durham and N. Flournoy, Random Walks for Quantile Estimation, Springer, New York, NY, USA, 1994.

[3] E. L. Korn, D. Midthune, T. T. Chen, L. V. Rubinstein, M. C. Christian, and R. M. Simon, “A comparison of two phase I trial designs," Statistics in Medicine, vol. 13, no. 18, pp. 1799-1806, 1994.

[4] J. Whitehead, "Bayesian decision procedures with application to dose-finding studies," International Journal of Pharmaceutical Medicine, vol. 11, no. 4, pp. 201-208, 1997.

[5] J. Babb, A. Rogatko, and S. Zacks, "Cancer Phase I clinical Trials: efficient dose escalation with overdose control," Statistics in Medicine, vol. 17, pp. 1103-1120, 1998.

[6] M. Gasparini and J. Eisele, "A curve-free method for phase I clinical trials," Biometrics, vol. 56, no. 2, pp. 609-615, 2000.

[7] S. Mukhopadhyay, "Bayesian nonparametric inference on the dose level with specified response rate," Biometrics, vol. 56, no. 1, pp. 220-226, 2000.

[8] L. M. Haines, I. Perevozskaya, and W. F. Rosenberger, "Bayesian optimal designs for phase I clinical trials," Biometrics, vol. 59, no. 3, pp. 591-600, 2003.

[9] N. Ting, Dose Finding in Drug Development, Springer, New York, NY, USA, 1st edition, 2006.

[10] S. Chevret, Statistical Methods for Dose-Finding Experiments, John Wiley \& Sons, Chichester, UK, 2006.

[11] G. Decoster, G. Stein, and E. E. Holdener, "Original article: responses and toxic deaths in Phase I clinical trials," Annals of Oncology, vol. 1, no. 3, pp. 175-181, 1990.

[12] M. J. Ratain, R. Mick, R. L. Schilsky, and M. Siegler, "Statistical and ethical issues in the design and conduct of phase I and II clinical trials of new anticancer agents," Journal of the National Cancer Institute, vol. 85, no. 20, pp. 1637-1643, 1993.

[13] M. J. Ratain, R. Mick, L. Janisch et al., "Individualized dosing of amonafide based on a pharmacodynamic model incorporating acetylator phenotype and gender," Pharmacogenetics, vol. 6, no. 1, pp. 93-101, 1996.

[14] D. R. Newell, "Pharmacologically based phase I trials in cancer chemotherapy," Hematology, vol. 8, pp. 257-275, 1994.

[15] J. O'Quigley, L. Z. Shen, and A. Gamst, "Two-sample continual reassessment method," Journal of Biopharmaceutical Statistics, vol. 9, no. 1, pp. 17-44, 1999.

[16] J. O'Quigley and X. Paoletti, "Continual reassessment method for ordered groups," Biometrics, vol. 59, no. 2, pp. 430-440, 2003.

[17] J. S. Babb and A. Rogatko, "Patient specific dosing in a cancer phase I clinical trial," Statistics in Medicine, vol. 20, no. 14, pp. 2079-2090, 2001.

[18] M. Tighiouart, A. Rogatko, and J. S. Babb, "Flexible Bayesian methods for cancer phase I clinical trials. Dose escalation with overdose control," Statistics in Medicine, vol. 24, no. 14, pp. 2183-2196, 2005. 


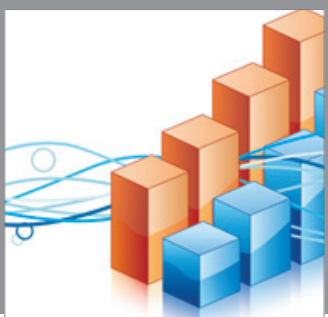

Advances in

Operations Research

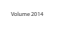

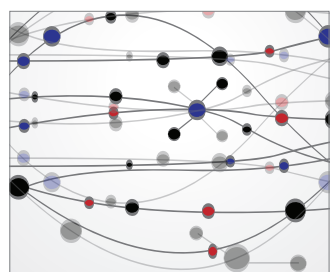

\section{The Scientific} World Journal
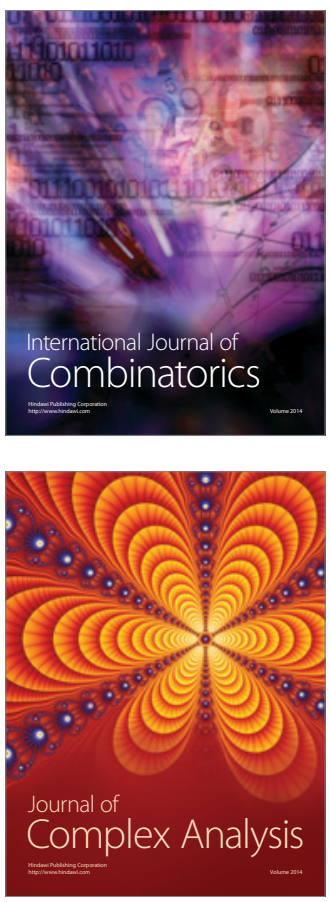

International Journal of

Mathematics and

Mathematical

Sciences
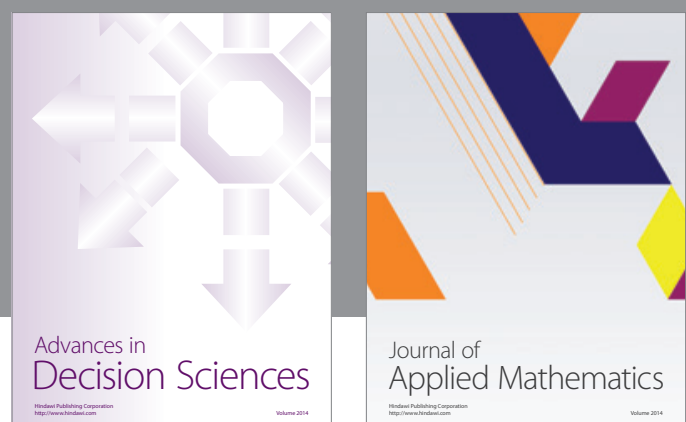

Journal of

Applied Mathematics
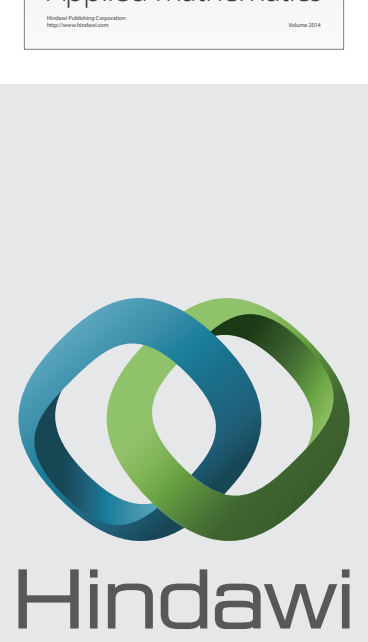

Submit your manuscripts at http://www.hindawi.com
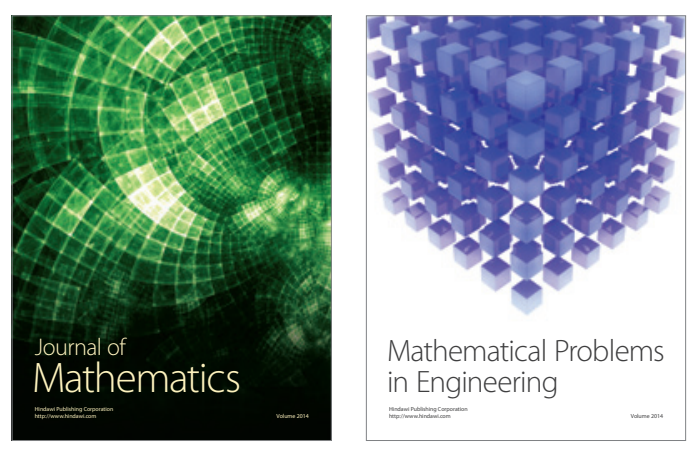

Mathematical Problems in Engineering
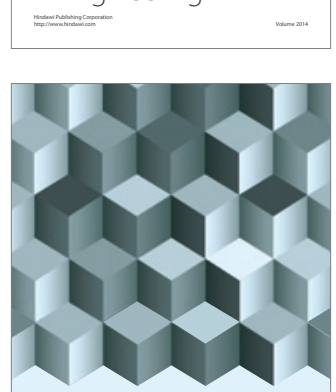

Journal of

Function Spaces
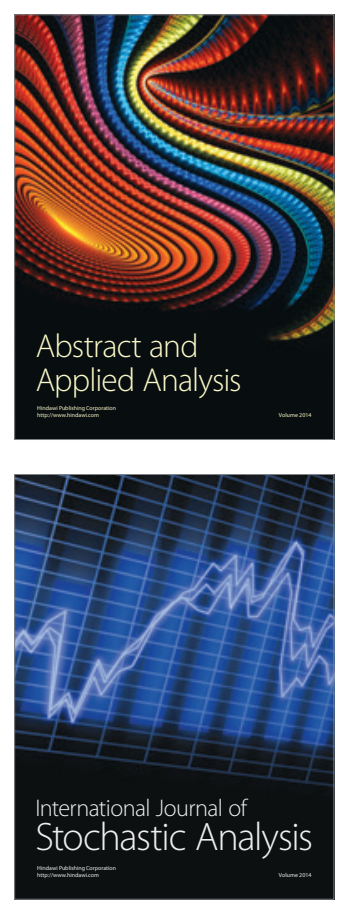

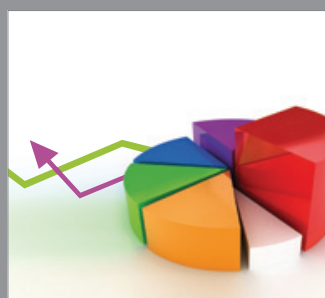

ournal of

Probability and Statistics

Promensencen
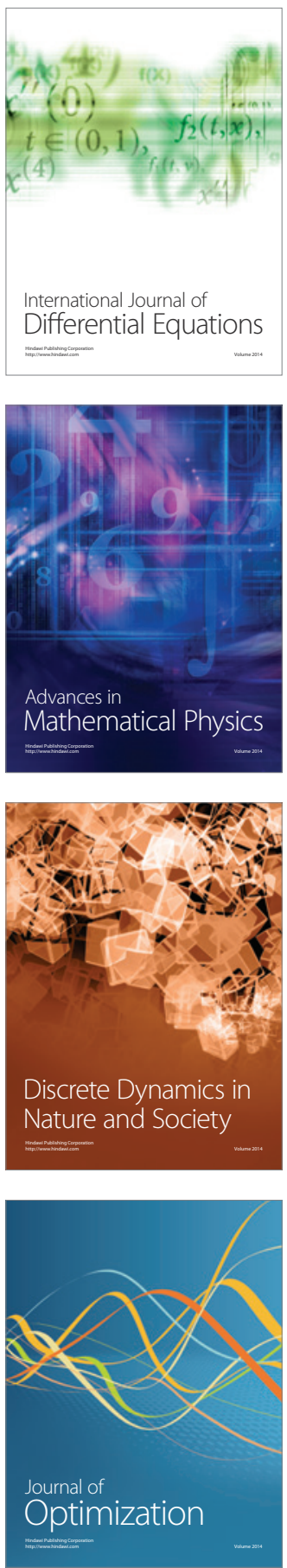